\title{
Estresse em Estudantes ao longo da Graduação Médica
}

\author{
Stress in Undergraduate Medical Students
}

\author{
Suzana Xui Liu Kam \\ Ana Luiza Siqueira de Toledo \\ Carla Colombo Pacheco \\ Giovanna Fernandes Borges de Souza ${ }^{I}$ \\ Victória Linhares Maia Santanal \\ Bruno Bonfá-Araujo ${ }^{I !}$ \\ Cássia Regina da Silva Neves Custódio ${ }^{I}$
}

\section{PALAVRAS-CHAVE}

- Educação Médica.

- Estresse Psicológico.

- Medicina.

\footnotetext{
Iniversidade de Mogi das Cruzes, Mogi das Cruzes, São Paulo, Brasil.

"Iniversidade São Francisco, Campinas, São Paulo, Brasil.
} 


\section{KEY-WORDS}

- Medical Education.

- Psychological Stress.

- Medicine.

Recebido em 27/5/19

Aceito em 29/6/19

\section{ABSTRACT}

Stress corresponds to physical, psychological and hormonal responses that occur when the body needs to adapt to a challenging situation. It is one of the most recurrent responses in the academic world due to the exhaustive routine and dense content that demand responsibility and competitiveness from the students. In medical undergraduate schools, we add to these factors the student's concern to learn the content that was discussed in class and the pressure exerted by the students themselves, in addition to the demands of society. Given the relevance of the topic, this study aimed to evaluate the occurrence of stress during medical undergraduate school in students from a private university in the Alto Tietê region, in the state of São Paulo. Two research instruments, the Lipp Adult Stress Symptom Inventory (ISSL) and the Perceived Stress Scale (PSS), were applied to $n=420$ undergraduate students $(67.14 \%$ females) attending the basic (first and second years) and vocational cycles (third to sixth years), and all participants were volunteers. According to the PSS, higher levels of stress were observed in students from the first to the third years, when compared to students from the fourth to the sixth years of medical school. The ISSL demonstrated the occurrence of stress in 65\% of the students, of which $9.04 \%$ were in the "near exhaustion" phase and $0.95 \%$ in the "exhaustion" phase, which represent the highest levels of stress, with the occurrence of symptoms being more prevalent in female students. Among all the analyzed groups, students attending the first to the third years showed a higher stress index than the others, which may be associated to the distribution of curricular contents with the predominance of conceptual disciplines, as well as the process of transition from theoretical contents to practical activities. These results may be related to the students' adaptation to the university routine, to the courses taken and to the obligations that higher education demands.

\section{INTRODUÇÃO}

O estresse é considerado uma das maiores epidemias mundiais do século $X_{X I}{ }^{1}$. Ele corresponde a uma resposta física, psíquica e hormonal que ocorre quando o corpo necessita de adaptação frente a um evento que pode causar desequilíbrio à homeostase do indivíduo². Numa situação de estresse, observa-se que, além do componente físico, há o envolvimento de uma resposta emocional, uma vez que são acionadas diversas estruturas neuroanatômicas, como o sistema límbico, amígdala e hipotálamo, envolvidas no controle de reações de medo e raiva ${ }^{3}$. Esta ativação resulta na estimulação do sistema nervoso simpático e aumento da liberação de catecolaminas, que promovem diversas alterações sistêmicas, como aumento da frequência cardíaca e deslocamento do fluxo sanguíneo para os músculos esqueléticos ${ }^{4}$. Posteriormente, o indivíduo passa a apresentar sintomas como cansaço e desgaste físico, que podem resultar em exaustão quando já tiverem sido esgotadas todas as tentativas de recuperação da homeostase, tornando o organismo vulnerável ao aparecimento de doenças como depressão, úlcera gástrica e hipertensão arterial ${ }^{2,5}$.

Um dos ambientes que mais propicia a ocorrência de eventos estressores é o ambiente universitário, que impõe uma rotina exaustiva devido às responsabilidades e exigências constantes ${ }^{6,7}$. Na graduação em Medicina, tais exigências se iniciam antes do ingresso no curso, um dos mais concorridos do exame vestibular. Acadêmicos do curso de Medicina apresentam níveis médios de ansiedade mais elevados do que a população geral, e a ocorrência de quadros depressivos pode aumentar significativamente ao longo do primeiro ano universitário ${ }^{8}$.

Adicionalmente, observa-se que, durante a graduação, fatores como dificuldades em encontrar períodos de lazer e privação do convívio familiar, associados à dependência financeira podem contribuir para o desenvolvimento do estresse. Estas condições podem dificultar a capacidade de raciocínio e de memorização, além de afetar o interesse do estudante pelo aprendizado. Futuramente, estes aspectos poderão influenciar negativamente a sua formação, bem como a sua atuação profissional e o relacionamento com o paciente ${ }^{2}$.

Entende-se que a complexidade do currículo médico, associada às tentativas do estudante em compreendê-lo, implicará sacrifícios emocionais e pessoais ${ }^{9,10}$. Assim, condições como as exigências para aprovação nas disciplinas e, consequentemente, a perda de controle sobre a disponibilidade de 
tempo resultam em privação de horas de sono e de atividades de lazer ${ }^{11,12}$. Fadiga extrema pelas horas dedicadas ao estudo e autocobrança para corresponder às suas expectativas, assim como às dos familiares e da sociedade tornam os estudantes de Medicina um grupo que, em particular, apresenta taxas maiores de estresse, além de outros distúrbios emocionais, quando comparado à população em geral ${ }^{2}$.

Corroborando estes dados, estudos mostram que os acadêmicos têm dificuldade em se adaptar a situações estressantes, o que interfere no seu estilo de vida. Desta forma, muitos estudantes buscam alívio e equilíbrio emocional por meio do uso de substâncias psicoativas ${ }^{1,9}$. Entre os alunos de graduação na área da saúde, altos níveis de estresse são apresentados não apenas por alunos de Medicina; estudos anteriores relatam índices superiores a 50\% em estudantes de Enfermagem e Odontologia ${ }^{13,14}$. Assim, diante da relevância do tema, este estudo teve como objetivo realizar um levantamento dos níveis de estresse ao longo do curso médico em uma universidade do Alto Tietê no Estado de São Paulo.

\section{MÉTODO}

Trata-se de um estudo quantitativo de corte transversal. Foram utilizados como população-alvo estudantes regularmente matriculados no curso de Medicina de uma universidade do Alto Tietê no ano de 2017. Nesse período, estavam regularmente matriculados 539 alunos, sendo 67,9\% mulheres ( $\mathrm{n}=$ 366 ) e $32,1 \%$ homens $(n=173)$. Os alunos estavam distribuídos em seis períodos, que possuem, em média, 90 alunos por turma.

Com base no cálculo amostral, determinou-se a necessidade da participação de 420 indivíduos. Foram selecionados 70 alunos matriculados por período/turma para a realização de um estudo com margem de erro de 5,5\% e intervalo de confiança de $95 \%$. Os participantes da pesquisa foram distribuídos de maneira homogênea em grupos de 70 alunos por turma, sendo $67,4 \%$ do sexo feminino $(n=283)$ e $32,6 \%$ do sexo masculino $(n=137)$. Todos os indivíduos participaram de maneira voluntária da pesquisa e nenhum dos participantes abordados se recusou a participar do estudo. A Figura 1 apresenta de forma gráfica a população e a seleção amostral.

\section{MATERIAL}

Foram utilizados dois instrumentos para avaliar o construto estresse: o Inventário de Sintomas de Estresse para Adultos de Lipp (ISSL) ${ }^{15}$, validado por Lipp e Guevara ${ }^{16}$, por meio do qual se podem identificar sintomas de estresse dos indivíduos, bem como a sua respectiva fase (alerta, resistência, quase exaustão e exaustão); e a Escala de Estresse Percebido (PSS) ${ }^{17}$, um ins-

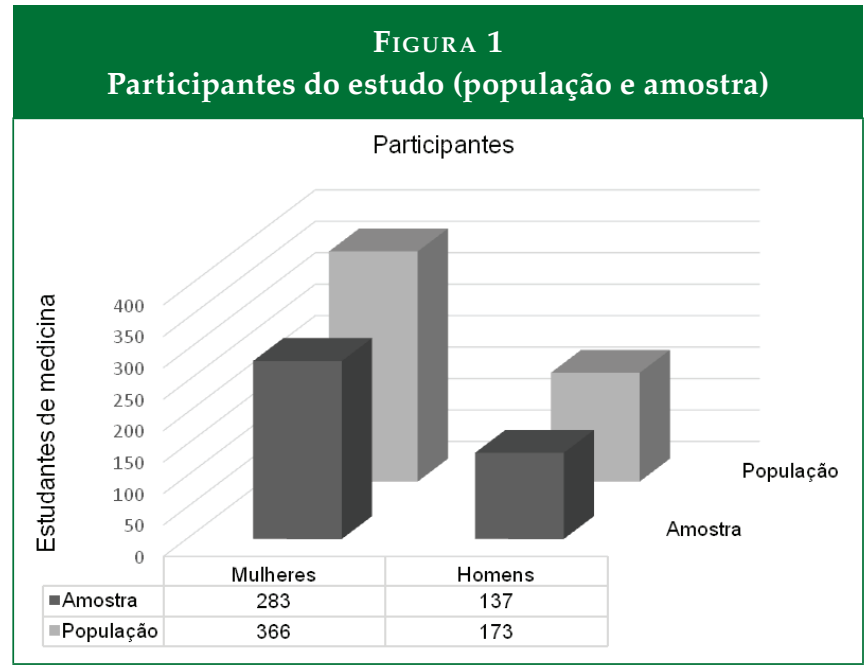

trumento com 14 questões cujas respostas apresentam um escore que varia de 0 a 4 . As questões que envolvem aspectos de percepção negativa $(1,2,3,8,11,12$ e 14) obedecem às seguintes pontuações: $0=$ nunca a $4=$ sempre. Por outro lado, as questões que envolvem fatores de percepção positiva $(4,5,6$, $7,9,10$ e 13) têm seus valores de pontuação invertidos, sendo que o 0 corresponde a 4 pontos, e assim sucessivamente. $\mathrm{O}$ valor total da escala corresponde à soma das pontuações das 14 questões, que resulta em escores que variam entre 0 e 56.

\section{PROCEDIMENTOS}

A pesquisa foi aprovada pelo Comitê de Ética em Pesquisa (CEP), com Parecer 1.602.723. Os estudantes foram abordados nas respectivas salas de aula após as devidas autorizações para a realização da pesquisa. Todos os participantes foram conscientizados acerca de seus riscos e benefícios, e aqueles que aceitaram participar voluntariamente da pesquisa assinaram o Termo de Consentimento Livre e Esclarecido (TCLE). Os questionários foram aplicados durante o ano letivo de 2017 nas salas de aulas dos estudantes, tendo sido excluídas da pesquisa as alunas pesquisadoras do presente estudo. Todas as análises estatísticas foram realizadas no software SPSS (Statistical Package for the Social Sciences). Os resultados dos instrumentos foram analisados por um psicólogo.

\section{ANÁLISE DE DADOS}

Na análise dos dados, usou-se a estatística descritiva para apresentação da composição amostral, e a correlação de Spearman foi empregada para identificar a relação entre o ISSL e o PSS. Utilizaram-se dois testes de normalidade com o objetivo de verificar se as variáveis apresentam distribuição normal ou gaussiana - Shapiro-Wilk e Lilliefor -, sendo que estes indicaram a 
não normalidade dos dados ( $\mathrm{p}<0,01)$. Após esta identificação, foi feita uma Análise de Regressão e em seguida foi realizada a Análise de Variância (Anova), com uso do teste post-hoc de Tuckey de comparação entre as médias, com o objetivo de identificar a diferença estatística do estresse entre as turmas estudadas.

\section{RESULTADOS}

O Inventário de Sintomas de Estresse para Adultos Lipp (ISSL) é classificado em quatro fases: "alerta", "resistência", "quase exaustão" e "exaustão". Cada fase representa um nível de estresse vivenciado, sendo que a primeira delas representa índices de estresse em que existe uma quebra da homeostase, enquanto as fases seguintes representam experiências mais intensas e estressoras, nas quais se nota que o indivíduo tem dificuldade em utilizar estratégias para controle do estresse ${ }^{15}$. A Tabela 1 retrata a dispersão em cada fase do instrumento, por ano.

Entre os 420 alunos pesquisados, 35\% não apresentaram estresse $(n=147)$, enquanto $65 \%(n=273)$ apresentaram algum sintoma característico desse quadro. A maior parte dos 273 estudantes (53,57\%) se encontrava na fase de "resistência", ao passo que 1,42\% apresentavam sintomas correspondentes à fase de "alerta"; 9,04\% estavam na fase de "quase exaustão"; e $0,95 \%$ na fase de "exaustão".

Quando analisados os dados de ocorrência de estresse de acordo com o sexo, observou-se que $66,43 \%$ dos alunos que apresentavam algum sintoma pertenciam ao sexo feminino. As mulheres também representaram 39,05\% dos alunos na fase de "resistência" e 7,62\% dos alunos incluídos na fase de "quase exaustão". A Figura 2 retrata a disposição dos sujeitos estudados em relação ao sexo de acordo com cada instrumento. Ambos os testes indicam maior prevalência de sintomas de estresse no sexo feminino.

Para a Escala de Estresse Percebido (PSS ${ }^{17}$,os parâmetros utilizados foram os seguintes: indivíduos com pontuação entre 0 e 14 foram considerados com nível de estresse "muito baixo", enquanto os que apresentaram valores entre 15 e 28 foram considerados como nível "baixo"; de 29 a 42, "moderado", ao passo que as faixas entre 43 e 56 são inseridas no estresse de nível "alto". Os resultados obtidos estão apresentados na Tabela 2.

É possível identificar que, de acordo com a PSS, 49,52\% dos alunos apresentaram resultados de estresse "moderado"; $42,62 \%$ se enquadravam em níveis "baixos"; 3,33\% em "muito baixo"; e 4,52\% em "alto".

Quando calculado o coeficiente de correlação de Spearman, verifica-se que, de acordo com cada resposta apresentada pelos estudantes, é possível identificar uma correlação significativa entre o ISSL e o PSS $(r=0,65 ; p=0,01)$. Estes dados indicam que, quanto maior o número de sintomas apresentados pelos estudantes de Medicina entrevistados (sintomas físicos e psicológicos, avaliados pelo ISSL), maior foi o estresse percebido por eles próprios (avaliado pelo PSS). Os dados também indicam que as mulheres apresentaram maiores índices de estresse, demonstrados em ambos os instrumentos quando realizado o teste $t$ de Student. Isto indica no ISSL que o sexo feminino $(\mu=15,12 ; D P=0,49)$ apresenta maior índice do que o sexo masculino $(\mu=11,20 ; D P=0,64)$, e essa diferença foi significativa $(p<0,001)$. No que diz respeito ao instrumento PSS, também foram encontradas diferenças significativas $(p<$ $0,001)$ no que concerne ao sexo, indicando que o sexo feminino

\begin{tabular}{|c|c|c|c|c|c|c|c|}
\hline Alunos & $1^{o}$ ano & $2^{\circ}$ ano & $3^{\circ}$ ano & $4^{\circ}$ ano & $5^{\circ}$ ano & $6^{\circ}$ ano & Total \\
\hline Sem estresse & 5,48 & 4,29 & 3,57 & 5,48 & 6,19 & 10,00 & 35,00 \\
\hline$n=$ & 23 & 18 & 15 & 23 & 26 & 42 & 147 \\
\hline Alerta & 0,48 & 0,48 & 0,24 & 0,24 & 0 & 0 & 1,43 \\
\hline$n=$ & 2 & 2 & 1 & 1 & 0 & 0 & 6 \\
\hline Resistência & 9,76 & 9,05 & 9,29 & 9,76 & 9,29 & 6,43 & 53,57 \\
\hline$n=$ & 41 & 38 & 39 & 41 & 39 & 27 & 225 \\
\hline Quase exaustão & 0,95 & 2,38 & 3,33 & 0,95 & 1,19 & 0,24 & 9,05 \\
\hline$n=$ & 4 & 10 & 14 & 4 & 5 & 1 & 38 \\
\hline Exaustão & 0,00 & 0,48 & 0,24 & 0,24 & 0 & 0 & 0,95 \\
\hline$n=$ & 0 & 2 & 1 & 1 & 0 & 0 & 4 \\
\hline Total & 16,67 & 16,67 & 16,67 & 16,67 & 16,67 & 16,67 & 100 \\
\hline$n=$ & 70 & 70 & 70 & 70 & 70 & 70 & 420 \\
\hline
\end{tabular}


Figura 2

Disposição dos níveis de estresse de acordo com instrumento e sexo
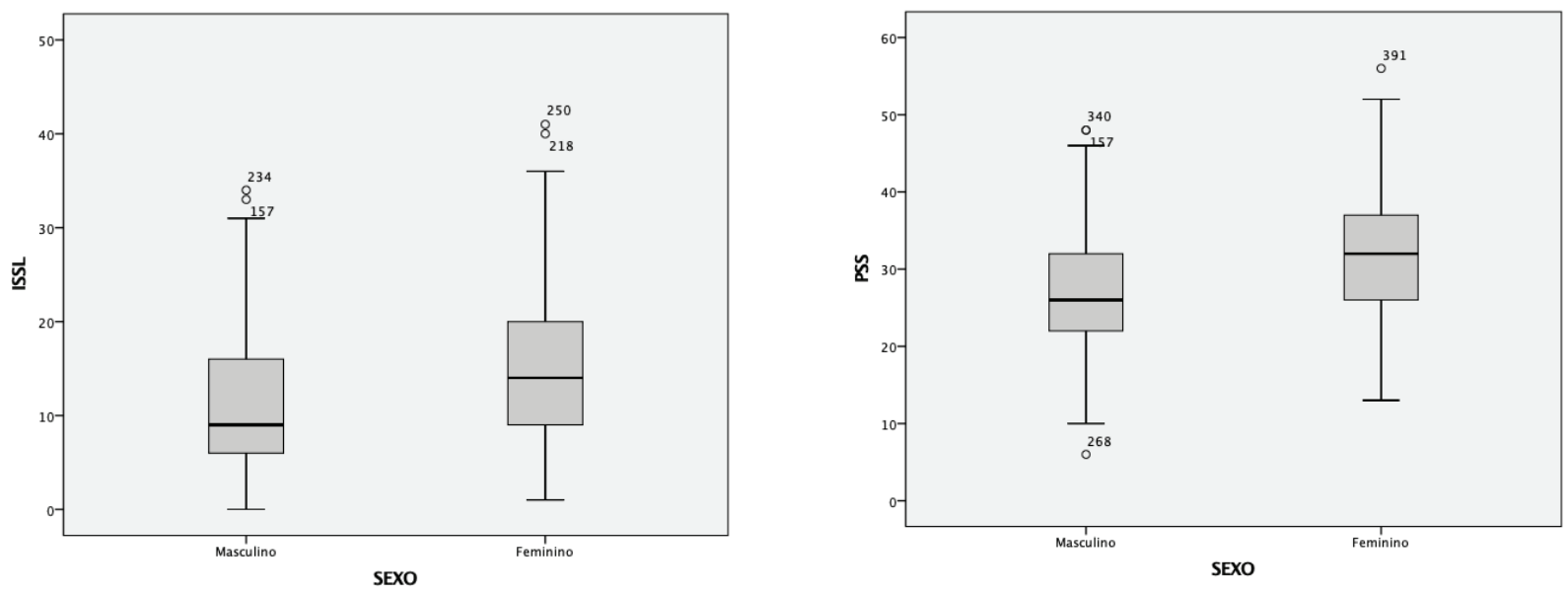

TABELA 2

Níveis de estresse dos alunos do primeiro ao sexto ano da graduação de acordo com a Escala de Estresse Percebido (PSS)

\begin{tabular}{|c|c|c|c|c|c|c|c|}
\hline Ano da graduação & $1^{o}$ ano & $2^{\circ}$ ano & $3^{\circ}$ ano & $4^{\circ}$ ano & $5^{\circ}$ ano & $6^{\circ}$ ano & Total \\
\hline Muito baixo & 0,24 & 0,24 & 0,48 & 0,71 & 0,71 & 0,95 & 3,33 \\
\hline$n=$ & 1 & 1 & 2 & 3 & 3 & 4 & 14 \\
\hline Baixo & 5,00 & 4,05 & 7,14 & 8,57 & 8,81 & 9,05 & 42,62 \\
\hline$n=$ & 21 & 17 & 30 & 36 & 37 & 38 & 179 \\
\hline Moderado & 11,19 & 10,48 & 8,10 & 6,90 & 6,43 & 6,43 & 49,52 \\
\hline$n=$ & 47 & 44 & 34 & 29 & 27 & 27 & 208 \\
\hline Alto & 0,24 & 1,90 & 0,95 & 0,48 & 0,71 & 0,24 & 4,52 \\
\hline$n=$ & 1 & 8 & 4 & 2 & 3 & 1 & 19 \\
\hline Total & 16,67 & 16,67 & 16,67 & 16,67 & 16,67 & 16,67 & 100 \\
\hline$n=$ & 70 & 70 & 70 & 70 & 70 & 70 & 420 \\
\hline
\end{tabular}

$(\mu=31,34 ; D P=0,44)$ apresenta também maior índice do que o sexo masculino $(\mu=26,92 ; D P=0,69)$.

Com o intuito de compreender a diferença entre os anos, foi realizada a análise de variância (Anova) e o teste de post-hoc de Tukey (Tabela 3), onde os subconjuntos indicam a separação feita por período cursado (primeiro ao terceiro ano; quarto ao sexto ano).

Tais achados indicam que existem diferenças significativas em função dos anos cursados. Para o instrumento ISSL, há uma diferença entre os períodos inicial e final de curso, indicando que o terceiro período apresenta maiores índices de estresse. Em relação ao PSS, os resultados apontam que o sexto ano apresenta menor índice de estresse percebido, enquanto o segundo ano apresenta maiores índices. No que diz respeito à distribuição das turmas e aos níveis de estresse, de acordo com a análise de regressão, foi encontrado que a classificação

\begin{tabular}{|c|c|c|c|c|c|c|c|}
\hline & $\begin{array}{r}\text { An } \\
N\end{array}$ & $\begin{array}{l}\text { va das } \\
\text { dicina }\end{array}$ & $\begin{array}{l}\text { TA B } \\
\text { irm } \\
\text { ara }\end{array}$ & $\begin{array}{l}\text { A } 3 \\
\text { s dos a } \\
\text { ISSL }\end{array}$ & $\begin{array}{l}\text { unos } \\
\text { o PSS }\end{array}$ & & \\
\hline \multirow{2}{*}{$\begin{array}{l}\text { Variável de } \\
\text { comparação }\end{array}$} & \multirow{2}{*}{$g l$} & \multirow{2}{*}{$F$} & \multirow{2}{*}{$n$} & \multirow{2}{*}{ Turma } & \multicolumn{3}{|c|}{ Subconjuntos } \\
\hline & & & & & 1 & 2 & 3 \\
\hline \multirow{6}{*}{ ISSL } & \multirow{6}{*}{5} & \multirow{6}{*}{$6,50^{* *}$} & 70 & $6^{\circ}$ ano & 10,73 & & \\
\hline & & & 70 & $5^{\circ}$ ano & 12,71 & & \\
\hline & & & 70 & $4^{\circ}$ ano & & 12,85 & \\
\hline & & & 70 & $1^{\circ}$ ano & & 13,27 & \\
\hline & & & 70 & $2^{\circ}$ ano & & & 16,23 \\
\hline & & & 70 & $3^{\circ}$ ano & & & 17,31 \\
\hline \multirow{6}{*}{ PSS } & \multirow{6}{*}{5} & \multirow{6}{*}{$6,60^{* *}$} & 70 & $6^{\circ}$ ano & 27,60 & & \\
\hline & & & 70 & $5^{\circ}$ ano & 27,89 & & \\
\hline & & & 70 & $4^{\circ}$ ano & & 28,37 & \\
\hline & & & 70 & $3^{\circ}$ ano & & 30,24 & \\
\hline & & & 70 & $1^{\circ}$ ano & & & 31,96 \\
\hline & & & 70 & $2^{\circ}$ ano & & & 33,43 \\
\hline
\end{tabular}


das turmas é responsável entre $5 \%$ (para o ISSL $-R^{2} 0,05$ ) e $6 \%$ (para o PSS $-R^{2} 0,06$ ) dos níveis de estresse. Ou seja, ao se verificar o valor estimado para a turma, percebe-se que a relação é inversamente proporcional, isto é, quanto maior o ano (exemplo: sexto ano), menor o índice de estresse.

\section{DISCUSSÃO}

Os resultados do presente estudo demonstraram que $65 \%$ dos estudantes do curso de Medicina apresentam algum nível de estresse. Estes achados estão em consonância com outros estudos realizados sobre o mesmo tema. Solansky e colaboradores $^{18}$ demonstraram que uma alta porcentagem de estudantes têm algum sintoma de estresse $(96,8 \%)$, sendo que, destes, $55,6 \%$ relataram experiências de estresse moderado, enquanto $41,2 \%$, estresse severo.

O presente estudo encontrou que 0,95\% dos estudantes analisados estavam na fase de exaustão e 4,52\% percebiam-se com altos níveis de estresse, taxas menores do que as do estudo de Solansky e colaboradores ${ }^{18}$. Entretanto, os autores destacaram que a presença de estresse considerado "severo" foi encontrada em estudantes que cursavam os anos finais de graduação, em contraposição aos resultados observados no presente estudo. Por outro lado, nossos achados confirmam os dados obtidos por Loureiro ${ }^{9}$, que observou que mulheres e estudantes do ciclo básico de um curso de Medicina se mostraram mais vulneráveis ao estresse do que os demais estudantes. Este resultado também está em consonância com nossa pesquisa, na qual foi possível identificar que $66,43 \%$ das alunas participantes apresentaram altos níveis de estresse segundo o ISSL.

Nossos resultados indicaram que estudantes que frequentavam os três primeiros anos do curso de Medicina apresentaram maiores índices nas fases mais intensas do estresse, enquanto estudantes que estavam cursando a metade final do curso apresentaram menores níveis de estresse, conforme indicado pela Tabela 3. Estes achados podem estar relacionados ao processo de adaptação dos alunos à rotina universitária e principalmente às disciplinas cursadas nos respectivos períodos letivos, uma vez que os dois primeiros anos da graduação médica são compostos pelo chamado "ciclo básico", que se caracteriza pelo predomínio de atividades teórico-práticas e menor parcela de conteúdos curriculares clínicos. O terceiro ano apresenta-se como a intersecção entre este ciclo básico e o designado "ciclo clínico", de caráter profissionalizante e que, de acordo com a Reforma Flexneriana, corresponderia aos quatros anos finais do curso ${ }^{19}$.

Segundo Dahlin et al. ${ }^{8}$, os estudantes do primeiro ano de graduação são os que estão submetidos a maior pressão nos estudos, devido à nova rotina enfrentada e às grandes exigências impostas a eles. Em nosso estudo, observamos que as frequências de alunos nas fases "sem estresse", "alerta" e "resistência" se mantiveram próximas do primeiro ao quinto ano, com números semelhantes de estudantes em cada classificação. Apenas na fase de "quase exaustão" foi observada uma diferença entre o segundo e o terceiro ano, com maior número de indivíduos apresentando sintomas específicos desta fase. O segundo e o terceiro ano da graduação apresentam uma matriz curricular com componentes curriculares mais densos, além de conteúdos teóricos extensos. Também há uma pressão para que todo o conteúdo conceitual dos três primeiros anos seja aprendido para a sua aplicação durante o ciclo clínico. Em decorrência destes fatores, os alunos tendem a ser mais exigentes quanto ao seu desempenho acadêmico durante os anos iniciais da graduação, o que pode ser um componente favorável ao aumento dos níveis de estresse nestas etapas. Um estudo realizado com alunos do curso de uma faculdade de Medicina situada na região do Vale do Paraíba (próxima ao Alto do Tietê, com características acadêmicas e geográficas similares às do local de coleta de dados do presente estudo) demonstrou que há uma correlação significativa entre as disciplinas cursadas nos primeiros anos do curso, suas respectivas notas e o aparecimento da síndrome de burnout nesses estudantes. Estes achados reforçam a hipótese de que as condições impostas nos anos iniciais de graduação podem desencadear um estado de esgotamento físico e mental que compromete acentuadamente o rendimento dos acadêmicos ${ }^{20}$.

Shaw e colaboradores ${ }^{1}$ descreveram os principais fatores estressores associados às duas principais etapas do curso de Medicina. Os autores sugerem que, durante o ciclo básico (designado basic science years), os principais problemas enfrentados são: dificuldade em manejar o tempo, solidão, ausência da família, ausência de atividades de lazer, adaptação aos novos colegas e competição pela obtenção das melhores notas. Por outro lado, na segunda metade do curso (denominado pelos autores clinical years), os pesquisadores consideram como fatores estressores: medo do fracasso, preocupação pelo aumento de atribuições e responsabilidades, desconforto ao realizar exames clínicos, temor de contaminação por doenças infectocontagiosas e afastamento dos colegas.

Alunos matriculados no quarto, quinto e sexto anos apresentam os menores níveis de estresse entre todos os anos estudados nesta pesquisa, resultados consonantes com os achados de Bassols e colaboradores ${ }^{21}$. Este achado pode ser explicado pela adaptação à rotina do curso médico, pela maior carga horária de disciplinas práticas ou pela euforia que acompanha a proximidade da conclusão do curso. Além disso, é possível 
que os níveis de estresse tenham diminuído porque o pico pode ter ocorrido durante os primeiros anos do curso ${ }^{21}$. A variação observada nos níveis de estresse nas turmas estudadas pode ser explicada pelas disciplinas cursadas, uma vez que há maior aplicabilidade dos conteúdos aprendidos com a atuação profissional realística.

Levando em consideração todos os anos avaliados no presente estudo, percebe-se que os níveis de estresse se mantêm elevados ao longo de toda a graduação, o que pode se tornar prejudicial à saúde do profissional médico. Observamos que 53,57\% dos estudantes avaliados (independentemente do sexo) se encontram na fase de resistência. Segundo Lipp ${ }^{15}$, a fase de resistência se caracteriza pela tentativa do indivíduo de manejar os fatores estressores com o objetivo de manter a homeostase orgânica. Entretanto, a autora ressalta que, se os fatores estressores persistirem ou não forem minimizados, os indivíduos podem evoluir para a fase de exaustão, na qual há maior risco de desenvolver patologias diretamente relacionadas com o estresse, como depressão e doenças cardiovasculares, como hipertensão arterial, entre outras.

O ensino médico exige que os estudantes enfrentem situações diárias que causam desgaste físico e psicológico. Um estudante de Medicina estressado apresenta pior desempenho, associado a comportamentos de medo e culpa. Esta condição pode contribuir para o adoecimento e sensação de incapacidade. Os alunos são alvo de muitas cobranças e responsabilidades, e estas podem se refletir no bem-estar e na saúde do indivíduo, como passar por situações de privação de sono, falta de lazer e de atividade física - fatores que alteram sua condição psicológica e prejudicam seu desempenho profissional no futuro ${ }^{10,12}$. Estes aspectos podem interferir diretamente na relação médico-paciente - uma vez que um médico que não esteja saudável física e mentalmente ficará impossibilitado de desempenhar com maestria suas atribuições devido ao comprometimento do raciocínio e da memorização - e no processo de aprendizagem ${ }^{2}$.

Embora os resultados aqui encontrados pareçam contraditórios em relação a outro estudo de mesma temática ${ }^{18}$, cabe salientar que aquele foi realizado há mais de cinco anos. A evolução tecnológica e as novas formas de relacionamento e interação social podem ter modificado este perfil (estudos futuros devem buscar compreender melhor esta relação). Provavelmente, os estudantes desenvolveram estratégias diversificadas de adaptação ao estresse, o que não minimiza o estado de alerta, uma vez que este estudo indicou uma grande porcentagem de indivíduos em fase de resistência (independentemente do ano cursado). Assim, devem-se sempre promover estratégias e orientações para que os alunos em fases modera- das do estresse não desenvolvam sintomas mais preocupantes apresentados em estágios avançados do estresse.

Estes achados podem estar relacionados ao processo de adaptação dos estudantes à rotina universitária do curso, às disciplinas cursadas e às exigências que uma formação superior demanda. Uma das limitações deste estudo é o fato de que os participantes foram inquiridos em diferentes momentos do ano letivo em razão de oportunidade e disponibilidade para a realização da coleta em uma única vez para todas as turmas, havendo a possibilidade de variações nos níveis de estresse em períodos de atividades acadêmicas mais intensas, como os de provas.

\section{CONSIDERAÇÕES FINAIS}

Os alunos do primeiro, segundo e terceiro ano foram identificados com maior nível de estresse e em fases mais avançadas quando comparados aos demais estudantes. Destaca-se que o sexo feminino apresentou maior resultado para ambos os instrumentos, indicando que mulheres apresentam maior nível de estresse nesta amostra. A despeito disso, o estudo mostra que há uma porcentagem expressiva de alunos na fase de resistência e com níveis moderados de estresse durante a graduação.

Estes achados sugerem que há a necessidade de monitoramento permanente e apoio psicológico aos discentes por parte das instituições de ensino superior e também dos próprios alunos e de seus familiares, com o intuito de prevenir o aparecimento de quadros de maior gravidade, como depressão, síndrome de burnout, etilismo e abuso de substâncias psicoativas, principalmente nos períodos em que o estresse se mostrou mais agravado. Para futuros estudos, sugere-se identificar a relação entre ingresso na prática clínica e sintomas de estresse, além de determinar as estratégias de enfrentamento utilizadas pelos estudantes.

\section{REFERÊNCIAS}

1. Shaw DL, Wedding D, Zeldow PB, Diehl N. Special Problems of Medical Students: Cap 6. In Wedding D, eds. Behavior\& Medicine. 2006; chapter 6, 67-83.

2. Oliveira JCB, Carvalho LC, Figueredo PMV. O estresse dos estudantes universitários de enfermagem de uma instituição privada do Rio de Janeiro. Revista Presença 2015;1(2), 39-55.

3. Paulino CA, Prezotto AO, Frias AC, Bataglia PR, Aprile MR. Sintomas de estresse e tontura em estudantes de pós-graduação. Revista Equilíbrio Corporal e Saúde 2015;2(1), 15-26.

4. Rocha MCP, Martino MMF, Grassi-Kassisse DM, Souza AL. Estresse em enfermeiros: $\mathrm{O}$ uso do cortisol salivar no 
dia de trabalho e de folga. Revista da Escola de Enfermagem da USP 2013;47(5), 1187-1194.

5. Dometita RJB. Stress tolerance, coping, and help-seekingamongcollegestudents. 2014. Doutorado [Tese] - Saint Louis University

6. Guhur MDLP, Alberto RN, Carniatto N. Influências biológicas, psicológicas e sociais do vestibular na adolescência. Roteiro 2010;35(1), 115-138.

7. Silva CR. Ansiedade no meio escolar. Brasília 2011. Graduação [Monografia] - Universidade de Brasília.

8. Dahlin M, Nils J, Bo R. Stress and depression among medical students: A cross-sectional study. Medical education 2005;39(6), 594-604.

9. Loureiro E. A relação entre o stress e os estilos de vida nos estudantes de medicina. Revista Brasileira de Educação Médica 2008;21(3), 209-214.

10. Olmo NRG, Ferreira LF, Prado AD, Martins LC, Dedivitis RA. Percepção dos estudantes de medicina do primeiro e sexto ano quanto à qualidade de vida. Diagn Tratamento 2012;17(4), 157-161.

11. Oliveira EN. Prevalência de sintomas depressivos em estudantes de medicina da Universidade Federal da Bahia. Salvador 2013. Graduação [Monografia] - Universidade Federal da Bahia.

12. Harada BA, Faxina CF, Capelleto CM, Simões JC. Perfil psicológico do estudante de medicina. Revista do Médico Residente 2013;15(2), 93-101.

13. Nascimento MG, Silva TPS, Colares V. Fatores relacionados ao estresse entre universitários de odontologia: Revisão sistemática de literatura. Revista Interdisciplinar de Estudos em Saúde 2017; 6(2), 57-72.

14. Preto VA, Sailer GC, Pereira SS, Teixeira CC, Parmigiani RS. Investigação sobre estresse e fases do estresse em universitários de enfermagem. 8 Simpósio em Saúde 2017; 6, 53.

15. Lipp MEN. Inventário de sintomas de stress para adultos Lipp (ISSL). São Paulo: Casa do Psicoólogo 2005.

16. Lipp MEN, Guevara AJH. Validação empírica do inventário de sintomas de stress (ISS). Estudos de Psicologia 1994;11(3), 43-49.

17. Luft CDB, Sanches SO, Mazo GZ, Andrade A. Versão brasileira da Escala de Estresse Percebido: Tradução e validação para idosos. Revista de Saúde Pública 2007;41(4), 606-615.

18. Solansky P, Desai B, Kavishwar A, Kantharia SL. Study of psychological stress among undergraduate medical stu- dents of government medical college. International Journal of Medical Science and Public Health 2012;1(2), 38-42.

19. Neves NMBC, Neves FBCS, Bitencourt ALGV. O ensino médico no Brasil: Origens e transformações. Gazeta Médica da Bahia 2008;75(2).

20. Mori MO, Valente TCO, Nascimento, LFC. Síndrome de Burnout e rendimento acadêmico em estudantes da primeira à quarta série de um curso de gradução em medicina. Revista Brasileira de Educação Médica 2012;36(4), 536-540.

21. Bassols AMS, Carneiro BB, Guimarães GC, Okabayashi LMS, Carvalho FG, Silva AB, Cortes GN, Rohde LAP, Eizirik CL. Stress and coping in a sample of medical students in Brazil. Arch. Clin. Psychiatry 2015;42(1), 1-5.

\section{CONTRIBUIÇÃO DOS AUTORES}

Suzana Xui Liu Kam: Participou da proposta do tema, definiu e redigiu os materiais e metodologia, objetivos da pesquisa, elaborou as tabelas presentes nos resultados e discussão, referências bibliográficas e revisão do resumo; Ana Luiza Siqueira de Toledo: Participou da introdução e discussão, e redigiu em forma de texto os resultados obtidos; Carla Colombo Pacheco: Participou da elaboração das tabelas presentes nos resultados e discussão, revisão do resumo e revisão das referências bibliográficas; Giovanna Fernandes Borges de Souza: Participou da elaboração dos resultados e discussão, redigiu o abstract, bem como revisão do resumo e escolha do título do texto; Victória Linhares Maia Santana: Participou da construção introdução e discussão, bem como da revisão do texto; Bruno Bonfá-Araujo: Realizou as análises e interpretação dos dados, e participou da redação e revisão do texto; Cássia Regina da Silva Neves Custódio: Orientadora principal, participou da elaboração do projeto, interpretação dos dados, redação e revisão do texto.

\section{CONFLITO DE INTERESSES}

Não há conflito de interesses de qualquer dos autores.

\section{ENDEREÇO PARA CORRESPONDÊNCIA}

Universidade de Mogi das Cruzes, Avenida Doutor Cândido Xavier de Almeida Souza, 200 - Centro Cívico, Mogi das Cruzes, CEP 08780-911, São Paulo, Brasil Tel.: (11) 4798-7000; FAX: (11) 4799-5233. 